\title{
Glucosylceramide in the Nervous System - A Mini-Review*
}

\author{
Norman S. Radin ${ }^{1,2}$
}

Accepted: September 10, 1993

A reviewer of this manuscript has recommended that I warn the reader that the hypotheses offered here do not have enough experimental support to make them widely accepted.

KEY WORDS: Glucosylccramide; myelin; cerebrosides.

\section{EVOLUTION AND THE CEREBROSIDES}

If, millions of years ago, a mutation had not appeared allowing animals to synthesize galactosylceramide (GalCer), our nervous systems might now be incapable of celebrating Bernard Agranoff's life and career. The lower animals, the protosomes, used glucosylceramide (GlcCer) to make a kind of myelin for their nervous system. A branch point appeared with the deuterostomes, who could now make myelin containing, as its major components, GalCer, GalCer sulfate, and GalCer made from 2-hydroxy fatty acids (1). The appearance of GalCer probably played a vital role in the brain's ability to make the efficient myelin that is needed for higher intelligence. In addition, the occurrence of GalCer in mammalian brain at a high concentration made it possible for neurochemists with a lipoidal bent to explore sphingolipid chemistry in great detail.

The enzyme systems involved in these syntheses are shown in Fig. 1. It is not yet clear how the hydroxyl group enters the acylamino position of ceramide but it is clear that hydroxy ceramide is a far better substrate than nonhydroxy ceramide for galactosylation. My

\footnotetext{
${ }^{1}$ Nephrology Division, Department of Intemal Medicine, and the Menta! Health Research Institute, University of Michigan, Ann Arbor, Michigan 48109-0676.

${ }^{2}$ Address reprint requests to: Dr. Norman S. Radin, The University of Michigan, Division of Nephrology - MSRB II, 1150 W. Medical Center Drive, Ann Arbor, MI 48109-0676.

* Special issue dedicated to Dr. Bernard W. Agranoff.
}

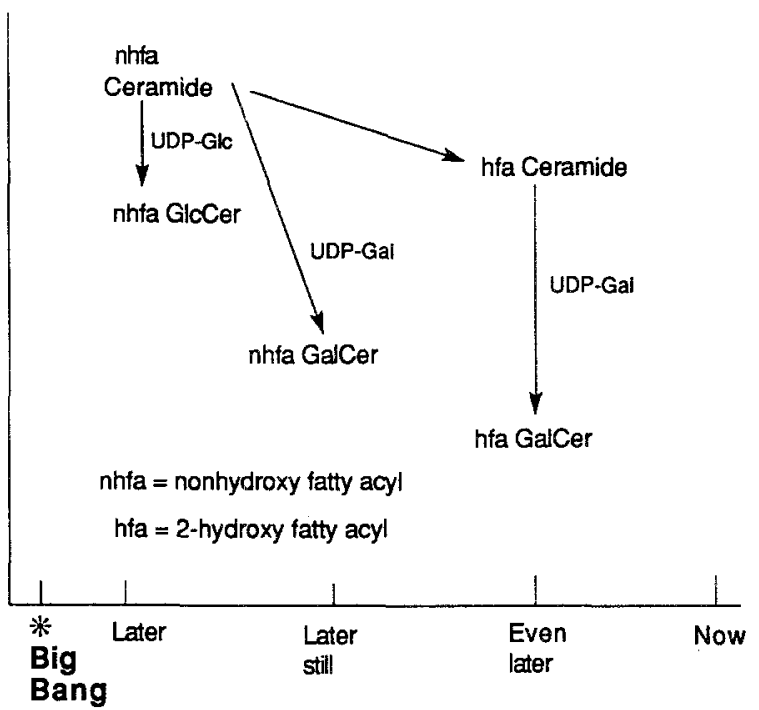

Fig. 1. Proposed evolutionary origin of GalCer and glial glycolipid synthesis.

drawing offers the possibility that nonhydroxy GalCer is made by a different, less active galactosyltransferase that may have formed earlier, historically, than the enzyme that utilizes hydroxy ceramide. To summarize, I propose that the initial mutation occurred in ceramide:UDP-glc glucosyltransferase, allowing the new enzyme to make nonhydroxy GalCer. A later mutation then converted this enzyme to one forming hydroxy GalCer. 
If the enzyme utilizing nonhydroxy ceramide is indeed closely related to glucosyltransferase, it might be sensitive to the same inhibitor that blocks glucosyltransferase. A comparison of the three synthases with PDMP (1-phenyl-2-decanoylamino-3-morpholino-1-propanol) could be illuminating. Other tests of this question do not appear to have been published, probably because of the somewhat greater cost of the labeled UDP-galactose needed for the assay of nonhydroxy GalCer synthase.

An alternative possibility, that psychosine (galactosyl sphingosine) is an intermediate in the synthesis of nonhydroxy GalCer, has some plausibility. Neskovic has reported that two different galactosyltransferases are involved; that is, partially purified GalCer synthase cannot galactosylate sphingosine (2). We now know that free sphingosine and psychosine normally exist in tissues, so psychosine synthase may be my hypothetical second evolutionary product (Fig. 1). Some laboratories have reported evidence for an enzyme that utilizes psychosine and fatty acid or fatty acyl CoA to produce nonhydroxy GalCer. For example, Curtino and Caputto found that microsomal fragments from chick brain could couple stearoyl-CoA and psychosine (3). The acylation went at $63 \%$ of full activity even when the microsomes were omitted, and it might be mentioned that the isolation of the cerebroside was preceded by a heating step with added methanol, $20 \mathrm{~min}$ at $55^{\circ} \mathrm{C}$. Acyl- $\mathrm{CoA}$ compounds possess a high-energy bond that can readily produce acylation of amines. Boiled microsomes did not produce any GalCer, possibly because the boiled tissue bound all the CoA ester and/or the psychosine.

A more recent paper (4) showed that labeled fatty acids, with unlabeled psychosine, could be converted to GalCer by intact neuroblastoma cells. Because the labeled GalCer did not contain labeled hydroxy acids or fatty acids longer in chain length than the original acid, it was suggested that the synthesis was enzymatically catalyzed. However, the failure of cells to hydroxylate or elongate the fatty acids is not a proof that the acylation of psychosine is enzymatic. The observed phenomenon could be attributed to enzymatic condensation of the labeled fatty acid with coenzyme $\mathrm{A}$ and subsequent nonenzymatic condensation with the amine. However, as the authors point out, the failure of labeled myristate, arachidonate, and lignocerate to react with psychosinedespite their conversion to CoA esters-suggests that the reaction is indeed enzymatic and curiously specific. Lignoceric acid is a typical component of nonhydroxy GalCer, yet it did not form GalCer. Probably the only way to come to a firm conclusion is to purify the putative condensing enzyme, as well as the galactosyltransferase acting on nonhydroxy ceramide.

\section{Glia versus Neurons}

The modern brain-like all organs, probably-still makes the prehistoric glycolipid, GlcCer, as well as its anabolic products. These products, which can be called glucosphingolipids (GSLs), include the nonionic derivatives possessing many sugar residues, the gangliosides possessing sialic acids, and the adhesion molecules possessing glucuronic acid and sulfuric acid moieties.

Not only have the oligodendroglia achieved the ability to make GalCer, but they seem also to have lost the ability to make GlcCer and some of its anabolites. Evidence for this conclusion came first from a study based on bulk-isolated cells from rat brain (5), which were assayed for their ability to synthesize the two cerebrosides. The neuronal preparations could make both cerebrosides, an observation consistent with the finding of both gluco- and galactolipids in neurons. GalCer synthesis was not much slower than in oligodendroglial preparations and GlcCer synthesis was somewhat faster than in whole homogenates. However no glucosyltransferase activity at all could be detected in the glial cells.

This finding might be artefactual in view of the extensive handling required for bulk isolation of the purified cells, as well as previous observations that glia and white matter contain GSLs. However both sugar transferases are located (in part, at least) in the Golgi membranes, which should probably survive the cell fractionation techniques. The most likely explanation for this contradiction is that glia obtain their GSLs by transfer from adjacent neurons or extracellular fluid. This view is supported also by later work from the Ledeen laboratory. Such uptake has been amply demonstrated with a variety of GSLs, which were shown to undergo metabolic conversions by the cells. A recent example is a study of cultured astrocytes, which were able to take up ganglioside GM1 from the medium and convert it to hydrolytic and sialylation products (6).

The release, or shedding, of GSLs from cells also appears to be a common phenomenon. An example comes from a study of cultured human neuroblastoma cells, which secreted $0.5 \%$ of their total gangliosides per hour (7). The other potential source of glial GSLs, extracellular fluid, is now well known to contain GlcCer and higher GSLs, bound to plasma lipoproteins.

There are, in addition, distinct proteins with the ability to transport GlcCer although they have not been demonstrated to occur between adjacent cells (8). Recently the saposins (heat-stable proteins involved in sphingolipid hydrolysis) have also been found to have the ability to accelerate the uptake of GSLs by membranes (9). A similar kind of evidence was obtained with 
cultured glial cells and antibody to 9-O-acetyl gangliosides (10). These cells failed to stain with the antibody but cells that were co-cultured with neurons (which react with the antibodies) stained quite well. This suggests transfer of the GSL from the nearby neurons. Thus it appears that the curious inability of glia to make their own GlcCer de novo, relying instead on direct uptake of GlcCer or on hydrolysis of higher GSLs, is a real characteristic of these cells.

Not only do glia lack glucosyltransferase but also the acetylgalactosaminyltransferase that makes the GSL, ganglioside GM2 (11). Neurons contain the enzyme and could serve as the source of this lipid. The authors suggest that the cells could obtain the lipid by phagocytosis of nerve cell debris as well as by transfer.

An apparent contradiction to the above observations is the report that cultured C6 glioma cells can make gangliosides and neutral GSLs from labeled serine (12). Serine, with respect to lipid metabolism, is a rather specific precursor of sphinganine, ending up in all GSLs, including GlcCer. Under the conditions of culture it would seem that contamination with neurons could not explain this synthetic process. In addition, other glial cell lines were found to have the ability to make GlcCer (13). These cells are cancer cells, and it is intriguing to think that mutational unveiling of the gene for making glucosyltransferase leads to uncontrolled growth and other tumor characteristics. Yet normal cells also make GlcCer and do not show cancerous properties. Perhaps glioma cells are abnormal also in lacking a controlling factor, such as glucocerebrosidase.

Glia arise from precursor cells; perhaps the precursor cells can make GlcCer and a critical change of some sort shuts down the transferase gene, leaving only the analogous galactosyltransferase gene. This step might be crucial to the transition to a committed glial cell.

\section{Why the Need to Stop Making GlcCer?}

If it is true that normal glia cannot make GlcCer, we can ask: what is the evolutionary survival value of the glial deficiency? Perhaps these cells, which must make relatively very large amounts of $\mathrm{GalCer}$, need to use every bit of available nucleotide sugar for this purpose. UDP-glc, once formed, is converted by epimerase to UDP-gal, the precursor of GalCer. The normal level of the two nucleotide sugars may not be high enough to saturate both cerebroside synthases and the rates of sugar transfer may be limited by the cellular concentration of nucleotide sugars. The same factors may explain why glia lack acetylgalactosaminyltransferase too; this enzyme utilizes UDP-galNAc, the synthesis of which must compete for the synthesis of UDP-gal. It would be interesting to see if glia also lack other enzymes that utilize UDP-sugars.

Some evidence to support this hypothesis was obtained in the case of GlcCer synthase of kidney (14). The $\mathrm{K}_{\mathrm{m}}$ for the binding of UDP-glc by ceramide:UDPglc glucosyltransferase in rats was found to be $250 \mu \mathrm{M}$ and the actual concentration of the nucleotide sugar in normal kidney was $\sim 150 \mu \mathrm{M}$, so the rate of GlcCer synthesis in this organ appears to be very sensitive to the nucleotide's concentration. (The glucosyltransferase occurs on the cytoplasmic face of Golgi membranes and the nucleotide sugar is present primarily or entirely in the cytoplasm, making the comparison a legitimate one.) In rats rendered diabetic, with blood glucose level uncontrolled by insulin, the UDP-glc concentration was $\sim 240 \mu \mathrm{M}$. Thus, if the ceramide concentration is not too low, and if the in vitro assays mirror the in vivo situation, we can assume that diabetic kidney makes GlcCer faster than normal. Treatment with insulin lowered the UDP-glc concentration only part way to normal.

In a study of GalCer synthesis, the $\mathrm{K}_{\mathrm{m}}$ for UDP. gal in rat brain microsomes was found to be $210 \mu \mathrm{M}$ (15), a value very similar to that found for glucosyltransferase in kidney. Thus it is likely that the same ratelimiting considerations apply to GalCer synthase in brain glia. In addition, brain is an unusually glucose-hungry organ, so biosynthetic reactions here may be especially sensitive to glucose availability.

What other physiological factors control GlcCer synthesis? The incubation of brain microsomes with gangliosides, in the assay for GlcCer synthase, showed that they are moderately effective inhibitors (16). The most effective gangliosides were the ones with the most sugar residues; over $50 \%$ inhibition was produced by $40 \mu \mathrm{M}$ GD1a. Some evidence was provided for the formation of a complex between the enzyme and the ganglioside. In one test, brain microsomes were incubated $10 \mathrm{~min}$ with $50 \mu \mathrm{M}$ GD1a, then diluted 10 -fold and centrifuged to remove unbound ganglioside. These membranes now showed 56\% lower synthase activity, a sign that the GSL had sedimented with the membranes.

These findings offer an explanation for the reports that patients with certain kinds of neurologicai damage can be aided in their recovery by injection of gangliosides. If these GSLs penetrate the brain to a sufficient concentration, they could block GlcCer synthesis by neurons and thus deplete the oligodendroglia of their GSLs. There is evidence implicating excessive glial growth during neural recovery; that is, glial regrowth may block axonal growth (in mammals). In a test with mice (16), the animals were injected i.p. daily for 5 days with 50 
$\mathrm{mg} / \mathrm{kg}$ of mixed brain gangliosides, then killed $3 \mathrm{~h}$ after the last injection. Brain and liver were homogenized, then assayed for GlcCer synthase activity. They were found to be significantly lower than the controls $(11 \%$ in brain, $27 \%$ in liver). In actual clinical therapeutic tests, much longer periods of ganglioside treatment are used, possibly gradually depleting the glia of their GlcCer and higher GSLs.

If this is the correct explanation for the beneficial effects of ganglioside treatment, other means of slowing GlcCer synthesis might be more useful (see the comments on PDMP below).

\section{Glucosylceramide as a Growth Factor}

Steroidal hormones and lipoidal vitamins, even though they are not peptides, are well known growth factors and it is not odd to believe that GSLs can also act as growth factors. In the case of GlcCer, the best known evidence is seen in Gaucher patients, who suffer from a genetic error in their glucocerebrosidase (or its activator protein, a saposin). They therefore accumulate large amounts of the glycolipid. The spleen grows so much that it may destroy red and white cells at an excessive rate, causing anemia or leukopenia. The excessive growth can lead to necrotic breakdown in the spleen, perhaps because angiogenesis does not keep up with the tissue growth. The liver can also grow, apparently because it too normally destroys blood cells and cannot hydrolyze the GSLs down to the building blocks. Bone marrow, which probably has a high rate of GlcCer synthesis, needed for blood cell construction, also tends to grow. However, being immobilized within the skeletal bones, it ends up destroying and weakening structural bone.

Curiously, students of Gaucher disease have not considered the growth feature of the disorder and nothing is known about the effects of the GlcCer accumulation on the better-known growth factors.

Experimental evidence identifying GlcCer as a growth factor, or activator of a growth factor, has been uncovered. The first evidence came from a study in which $100 \mathrm{mg} / \mathrm{kg}$ of conduritol B epoxide (dehydroinositol) was injected i.p. into young growing mice once a day for eight days (17). This compound inactivates GlcCer $\beta$ glucosidase, causing accumulation of the GSL in various organs. At the end of this period, the brains were found to be significantly enlarged (13\%). Liver was also larger (9\%). Unfortunately the GlcCer content of the brains was not determined; the much higher content of GalCer in brain makes this analytical problem very difficult.

A recent publication, based on cyclophellitol, a newly discovered inhibitor of GlcCer glucosidase, did not report increases in organ weights (18). Here, the new inhibitor, which is somewhat more effective than conduritol $B$ epoxide, was injected at $17 \mathrm{mg} / \mathrm{kg}$ for 5 days and the animals were evaluated 2 days later. Perhaps the failure to find organ growth was the result of accumulating glucosylsphingosine, a toxic lyso-GSL that has been found in Gaucher tissue. If this explanation is correct, it shows that there is a delicate balance between the stimulatory and inhibitory effects of blocking GlcCer breakdown.

GlcCer glucosidase is inhibited by nucleic acids, including tRNA (19). As little as $2 \mu \mathrm{g} / \mathrm{ml}$ reduced the enzyme's activity by $35-70 \%$. Whether lysosomes are influenced by this phenomenon remains unclear, but lysosomal nucleases exist so there must be some nucleic acid in these particles. Phosphatidylserine, a natural stimulator of $\beta$-glucosidase, counteracted the effect of nucleic acids. It will be interesting to see if factors controlling the levels of free nucleic acids or the synthesis of phosphatidylserine mediate the effect of GlcCer on growth. Other polyacidic substances, such as chondroitin sulfate, can act as inhibitors of lysosomal hydrolases and probably also block (or stabilize) $\beta$-glucosidase.

A more direct attempt at stimulating growth by GlcCer was made in mice, using a suspension of GlcCer injected i.p. $(20,21)$. The lipid was partially emulsified with a relatively nontoxic nonionic detergent, Myrj 52 (polyoxyethylene partially esterified with stearic acid). Much of the lipid goes to the liver, overloading the hydrolytic capacity of the organ. The liver grew 18-24\% in just 1 or 2 days. Both the protein and DNA content rose similarly, so the GlcCer seems to have induced faster cell division and growth, not simple hydration. The specific activity of two enzymes closely related to proliferation also increased: thymidine kinase rose 46$73 \%$ and ornithine decarboxylase rose as much as 101\%. These changes would seem to indicate that GlcCer has real mitogenic properties, although the possibility was not ruled out that the growth was due to some kind of secondary effect. Histological examination of the liver ought to be illuminating.

In the experiment described earlier in this review (14), accelerated synthesis of GlcCer by the diabetic rats was associated with a significant increase $(14 \%)$ in kidney size. Injecting the inhibitor of GlcCer synthase, PDMP, into the rats with the enlarged kidneys brought the kidney weights down to normal without affecting the blood glucose levels. Thus the elevation in tissue UDPglc due to hyperglycemia had no effect on kidney size when it couldn't be utilized to glucosylate ceramide.

Control rats did not respond to PDMP-induced synthase blockage by shrinking their kidneys below normal; 
probably shrinkage would be observed if the enzyme blockage were to be prolonged. In the case of mice, PDMP given over a 12-day period did shrink the kidneys significantly (22).

Another study relating GlcCer levels to kidney size was carried out by injecting young male mice with testosterone acetate (23). Kidneys, especially in male mice, normally grow markedly with maturation; this is accompanied by increases in the level and excretion of GSLs. We found that the specific activity of GIcCer synthase also rises with age while the level of GlcCer glucosidase decreases with age. Both enzyme changes act to elevate the level of GlcCer. The androgen accelerated kidney growth, as noted in previous studies, and it also accelerated the normal changes in both enzymes. Thus the increase in GlcCer was accompanied by an increase in organ size. Estradiol, on the other hand, shrank the kidneys and greatly increased $\beta$-glucosidase. There was little effect on synthase specific activity but the changed hydrolase would be expected to lower GlcCer levels. Thus the estrogen and androgen compete with each other for control of GlcCer level and kidney size.

Extension of this study to actual mass analysis of GlcCer and its precursors, ceramide and UDP-glc, should yield interesting data.

Several studies with cultured cells have also reinforced the belief that GlcCer stimulates growth. For example, blocking GlcCer synthase of Madin-Darby canine kidney cells with PDMP or with acyl homologs of PDMP produced slowed growth (protein per dish) and thymidine incorporation into DNA (normalized on protein mass) (24). Fatty acyl chains of 6 to 18 carbon atoms were used to make the PDMP homologs. The longer the acyl chain, the greater was the inhibition of GlcCer synthesis, assayed in vitro with several types of cells. With mouse brain microsomes, the effectiveness of $20 \mu \mathrm{M}$ inhibitor ranged from 14 to $62 \%$ inhibition. The effectiveness against cell growth and DNA synthesis showed a comparable correlation, although the range of effects was much greater. Comparison of two of the inhibitors with regard to GlcCer levels also showed a positive correlation between loss of GlcCer and slowing of growth.

A report that the $B$ subunit of cholera toxin could stimulate thymocyte cell proliferation (25) was interpreted to signify that ganglioside GM1 (which binds the toxin strongly) is involved in the proliferative process. One could explain the effect as a removal of the normal inhibitory action of GM1 on GlcCer synthesis. The toxin was indeed found to block the inhibitory effect of GM1 on GlcCer synthase of brain membranes (16). It appears that the toxin has a higher affinity for GM1 than does the glucosyltransferase. This observation further strengthens the hypothesis that GlcCer is a mitogenic agent.

Whether GlcCer or a closely related metabolite is the mitogenic lipid is still unclear. A few tests with GalCer, which would be expected to yield ceramide (as would GlcCer), showed it to be rather inert. Unfortunately the actual ceramide level was not determined. The first anabolic product of GlcCer is lactosylceramide, which has indeed been found in some cell systems to act as a growth stimulator. The second anabolic product of GlcCer is the sialylated lactosylceramide, ganglioside GM3. This too has been found to have activity as a growth stimulator. However both GSLs are catabolized to form GlcCer and thus may really be active because of this reaction.

\section{Ceramide, the Lipoidal Precursor of GlcCer}

The ceramide can consist of a $\mathrm{C}_{16}$ to $\mathrm{C}_{24}$ fatty acid combined in amide linkage with a sphingol (sphingosine, sphinganine, phytosphingosine, or a longer or shorter homolog). Phytoceramide probably does not occur in the nervous system (since phytoGSLs have not been discovered there) while $\mathrm{C}_{20}$-sphingosine is relatively prominent in brain, where it acts as a precursor of the gangliosides. Ceramides containing nonhydroxy acids are readily detected in brain, but we have been unable to detect hydroxyl-containing ceramides (the major precursors of GalCer). Perhaps hydroxy ceramide is galactosylated so rapidly that it does not accumulate to a detectable level.

Research on ceramide biosynthesis has an interesting past. Yavin and Gatt found that acid ceramidase, the lysosomal hydrolase, acts readily in the reverse direction (26). However, most of this work was done with fatty acids that do not occur in GSLs, such as oleic acid. It appears likely that the driving force for this synthesis is the formation of a highly insoluble ceramide from a relatively soluble fatty acid. If instead one starts with a highly insoluble fatty acid, so typical of GSLs, this driving force is greatly diminished. Further evidence against this route for de novo synthesis comes from the observation that people with Farber's lipogranulomatosis, who suffer from a genetically inadequate amount of acid ceramidase activity, do not have any difficulty in making new ceramide molecules. The net overproduction accounts for the painful accumulation of ceramide.

(Incidentally, the extra ceramide found in patients with this disorder leads to greater synthesis of the GSLs, suggesting that the rate of GlcCer synthesis is normally restricted - not only by the availability of UDP-glc - but also by the availability of the lipoidal precursor. Some patients exhibit brownish skin, which is seen also in some Gaucher patients; this may mean an association of 
GIcCer with an iron-containing protein. Another feature of ceramidosis is the occasional finding of pebble-textured skin, possibly due to the now well-established function of ceramide and GlcCer in skin maturation and permeability.)

For a while it seemed that the synthetic capability of ceramidase could, as with other hydrolases with demonstrated reversible activity, be considered a curious irrelevance. However later work seems to have shown that it does occur in lysosomes. Okabe and Kishimoto (27), using doubly labeled ceramide injected into mouse brain, found that new ceramides were formed from the two moieties, apparently during the hydrolytic step. Rather than a net synthesis, this kind of biosynthesis is more of a recycling of valuable metabolites or a "reshaping" of the molecular species available for further ceramide anabolic reactions. Additional findings of this sort have come from the laboratories of Tettamanti and Sonnino, who have been making various doubly-labeled GSLs.

In 1966, Sribney reported the synthesis of ceramide from sphingosine and stearoyl-CoA in rat brain microsomes (28). This finding was confirmed and elaborated in a study using mouse brain microsomes, where it was shown that oleate was a poor acyl donor while stearate and lignocerate $\left(\mathrm{C}_{24}\right)$ were much better (29). This evidence pointed to ceramide synthase as the main factor controlling the distribution of the different fatty acyl groups in the GSLs. Curiously, sphinganine as a substrate was almost as reactive as sphingosine despite the relative rarity of sphinganine in the higher sphingolipids.

If sphinganine-containing ceramide is almost as good as unsaturated ceramide as an acceptor for glucose, there must be a sorting step prior to glucosylation that discriminates against the saturated substrate. (Sphinganine is a very minor component of the higher sphingolipids.) While one could propose an alternative explanation, that glucosidase hydrolyzes saturated GlcCer more rapidly than unsaturated GlcCer, this is improbable because hydrolases tend to be relatively nonspecific. One could also propose that ceramidase hydrolyzes saturated ceramide more rapidly than unsaturated ceramide, or that a substrate-transferring system discriminates between the two. The sorting step is probably due to the existence of a dehydrogenase that converts $\mathrm{N}$-acyl sphinganine to $\mathrm{N}$ acyl sphingosine (Fig. 2). This is the only clearly defined route for the formation of sphingosine. The original report to this effect (30) has been definitely confirmed by later reports. However, data supporting other routes of sphingosine synthesis look too good to disregard, so there may still be room for minor enzymes (31).

This dehydrogenation does not mean that ceramide is made only from sphinganine. Both kinds of ceramide

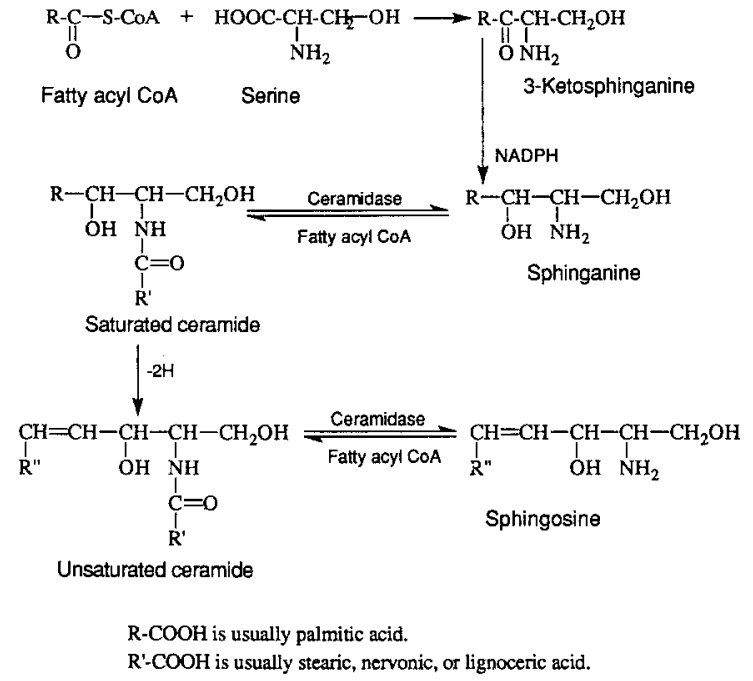

Fig. 2. Biochemical interrelationships between sphinganine, sphingosine, and ceramides.

undergo hydrolysis to form the free sphingols, which then are acylated by acyl-CoA esters. The free sphingols, mainly sphingosine, exist in tissues at low but measurable levels. Sphinganine apparently occurs as the free amine because it is synthesized de novo and also liberated by hydrolysis of saturated ceramide. Sphingosine, however, occurs only because it is liberated by ceramidase. To confirm the hypothesis that ceramide anabolism is the selective step, the actual sphingol distribution in tissue ceramides should be determined - are they low in sphinganine? This question is particularly important in view of recent findings that saturated and unsaturated ceramides produce different physiological effects.

A group of naturally occurring inhibitors of ceramide synthase, the fumonisins, has been discovered (32). They lead to accumulation of sphingols and loss of sphingolipids. Highly toxic effects are produced in vivo, including the formation of hepatic tumors. The latter effect could be due to lack of ceramide (see below for more on this).

A form of ceramidase active at a more alkaline $\mathrm{pH}$ deserves mention here but its effect is quite indistinct. It does not compensate for the lack of acid ceramidase in Farber's disease. It may play a special role, perhaps reshaping ceramide molecular species in a particular cellular site.

Acid ceramidase is inhibited by free sphingosine and fatty acid, the two products of its action. These products, in a living cell, may be removed from the lysosomes too quickly to interfere. A synthetic ceramide-like amide, N-oleoyl ethanolamine, has been de- 
scribed as being a "potent" inhibitor (33) and it would be worth testing other ethanolamides for possibly superior effectiveness. Evidence has been offered for the natural occurrence and hydrolysis of other ethanolamides (34). These compounds are formed by hydrolysis of Nacylated phosphatidylethanolamine and have physiological activity. Curiously, $\mathrm{N}$-arachidonoyl ethanolamine (anandamide) has been identified as the natural cannabinoid-like metabolite in brain (35). It is intriguing to think that anandamide or the other ethanolamides act by inhibiting ceramidase.

There is some evidence that ceramidase action requires an activator protein, although in vitro assays of the enzyme are invariably carried out in the presence of detergents and sodium cholate or taurocholate. This assay method probably obviates the need for an activator protein. A patient with a genetic defect, the inability to synthesize one of the saposins, was found to have a low level of ceramidase, as well as other hydrolases (36). Preliminary tests (Y. Kishimoto, personal communication) have shown that ceramidase action is stimulated by saposin $D$, one of the four saposins.

Some profoundly important effects of ceramide on growth and differentiation have recently been uncovered in HL-60 cells and it will be interesting to see how general these relationships are and whether GlcCer is involved in the mechanism of action. Several agents, such as 1,25-dihydroxyvitamin D3, tumor necrosis factor- $\alpha$, exogenous bacterial sphingomyelinase, and $\gamma$-interferon cause a rapid, temporary liberation of ceramide. The source of the lipid is believed to be sphingomyelin, which ordinarily occurs at a much higher concentration than GlcCer, and which seems to be hydrolyzed by an acid or neutral sphingomyelinase. Not yet ruled out, it seems to me, is the possibility that the ceramide, in some cases, is formed from sphingomyelin by reaction with diacylglycerol in a reverse reaction of the enzyme, sphingomyelin synthase (forming lecithin as well as ceramide). This enzyme, which simply catalyzes an exchange between two lipid moieties for attachment to a phosphocholine moeity, appears on the face of it to catalyze an easily reversed reaction.

Synthetic ceramides, such as $\mathrm{N}$-acetylsphingosine used at a low concentration, inhibit cell growth and induce differentiation (37). We too have found that a longer chain ceramide, octanoyl sphingosine, also inhibits cell division and growth (38).

An elevation in cellular ceramide level can also be produced in cultured cells by PDMP, suggesting that a significant portion of the ceramide pool is used for GlcCer synthesis. One might expect that most of the pool is used for making sphingomyelin, which occurs at a much higher concentration than GlcCer. Thus a blockage of the minor route would not be expected to produce much of a change in ceramide. Probably the explanation for the magnitude of the rise is that sphingomyelin turnover is relatively slow, compared to that of GlcCer. An alternative possibility is that PDMP inhibits sphingomyelin biosynthesis, so that the ceramide accumulation is due to blockage of both routes of ceramide utilization. However, including labeled palmitic acid in the culture medium of MDCK cells showed that PDMP almost doubled the conversion of the labeled acid to sphingomyelin, presumably by making ceramide more available (39). It would be worth determining whether some of the natural ceramide-elevating factors mentioned above induce their effects by slowing GicCer synthesis, as well as by speeding sphingomyelin breakdown.

\section{REFERENCES}

1. Kishimoto, Y. 1986. Phylogenetic development of myelin glycosphingolipids. Chem. Phys. Lipids 42:117-128.

2. Neskovic, N. M. Interactions of the purified ceramide galactosyltransferase with exogenous lipids and detergents. Abstract in Enzymes of Lipid Metabolism, NATO Advanced Research Workshop and CNRS-INSERM International Symposium Oct. 14, 1985.

3. Curtino, J. A., and Caputto, R. 1974. Enzymic synthesis of cerebroside from glycosylsphingosine and stearoyl-CoA by an embryonic chicken brain preparation. Biochem. Biophys. Res. Commun. 56:142-147.

4. Farrer, R. G., and Dawson, G. 1990. Acylation of exogenous glycosylsphingosines by intact neuroblastoma (NCB-20) cells. J. Biol. Chem. 265:22217-22222.

5. Radin, N. S., Brenkert, A., Arora, R. C., Sellinger, O. Z., and Flangas, A. L. 1972. Glial and neuronal localization of cerebroside-metabolizing enzymes. Brain Res. 39:163-169.

6. Masco, D., Flott, B., and Seifert, W. 1989. Astrocytes in cell culture incorporate GM1 ganglioside. Glia 2:231-240.

7. Li, R., and Ladisch, S. 1991. Shedding of human neuroblastoma gangliosides. Biochim. Biophys. Acta 1083:57-64.

8. Metz, R. J., and Radin, N. S. 1982. Purification and properties of a cerebroside transfer protein. J. Biol. Chem. 257:12901-12907.

9. Soeda, S., Hiraiwa, M., O'Brien, J. S., and Kishimoto, Y. 1993. Binding of cerebrosides and sulfatides to saposins A-D. J. Biol. Chem. 268:18519-18523.

10. Nebdez-Otero, R., and Constantine-Paton, M. 1990. Granule cell induction of 9-O-acetyl gangliosides on cerebellar glia in microcultures. Dev. Biol. 138:400-409.

11. Sbaschnig-Agler, M., Dreyfus, H., Norton, W. T., Sensenbrenner, M., Farooq, M., Byrne, M. C., and Ledeen, R. W. 1988. Gangliosides of cultured astroglia. Brain Res. 461:98-106.

12. Lin, Y., and Leskawa, K. C. 1992. Effects of the major metabolite of cocaine, benzoylecgonine, on reuronal and glial glycosphingolipid synthesis and viability. 1992. Glycobiology 2: abstract 7.02 .

13. Neskovic, N. M., Rebel, G., Harth, S., and Mandel, P. 1981. Biosynthesis of galactocerebrosides and glucocerebrosides in glial cell lines. J. Neurochem. 37:1363-1370.

14. Zador, I. Z., Deshmukh, G. D., Kunkel, R., Johnson, K., Radin, N. S., and Shayman, J. A. 1993. A role for glycosphingolipid accumulation in the renal hypertrophy of streptozotocin-induced diabetes mellitus. J. Clin. Invest. 91:797-803. 
15. Arora, R. C. and Radin, N. S, 1972. Synthetic inhibitors of galactocerebroside biosynthesis. Biochim. Biophys. Acta 270:254 259.

16. Shukla, G. S., Shukla, A., and Radin, N. S. 1991. Gangliosides inhibit glucosylceramide synthase: possible role in ganglioside therapy. J. Neurochem. 56:2125-2132.

17. Hara, A., and Radin, N. S. 1979. Enzymic effects of B-glucosidase destruction in mice: changes in glucuronidase levels. Biochim. Biophys. Acta 582:423-433.

18. Atsumi, S., Nosaka, C., Inuma, H., and Umezawa, K. 1992. Inhibition of glucocerebrosidase and induction of neural abnormality by cyclophellitol in mice. Arch. Biochem. Biophys. 297:362367.

19. Sano, A. and Radin, N. S. 1988. The inhibition of glucosylceramide $\beta$-glucosidase and other acid hydrolases by nucleic acids. Biochem. J. 254:297-300.

20. Datta, S. C., and Radin, N. S. 1986. Glucosylceramide and the level of the glucosidase-stimulating proteins. Lipids 21:702-709.

21. Datta, S. C., and Radin, N. S. 1988. Stimulation of liver growth and DNA synthesis by glucosylceramide. Lipids 23:508-510.

22. Inokuchi, J., Mason, I., and Radin, N. S. 1987. Antitumor activity in mice of an inhibitor of glycosphingolipid biosynthesis. Cancer Lett. 38:23-30.

23. Shukla, A., Shukla, G. S., and Radin, N. S. 1992. Control of kidney size by sex hormones; possible involvement of glucosylceramide. Amer. J. Physiol. 262:F24-F29.

24. Abe, A., Inokuchi, J., Jimbo, M., Shimeno, H., Nagamatsu, A., Shayman, J. A., Shukla, G. S., and Radin, N. S. 1992. Improved inhibitors of glucosylceramide synthesis. J. Biochem. 111:191196.

25. Spiegel, S., Fishman, P. H., and Weber, R. J. 1985. Direct evidence that endogenous GM1 ganglioside can mediate thymocyte proliferation. Science 230:1285-1287.

26. Yavin, E., and Gatt, S. 1969. Enzymatic hydrolysis of sphingolipids. VIII. Further purification and properties of rat brain ceramidase. Biochemistry 8:1692-1698.

27. Okabe, H., and Kishimoto, Y. 1977. In vivo metabolism of ceramides in rat brain. Fatty acid replacement and esterification of ceramide. J. Biol. Chem. 252:7068-7073.

28. Sribney, M. 1966. Enzymatic synthesis of ceramide. Biochim. Biophys. Acta 125:542-547.
29. Morell, P., and Radin, N. S. 1970 . Specificity in ceramide biosynthesis from long chain bases and various fatty acyl coenzyme A's by brain microsomes. J. Biol. Chem. 245:342-350.

30. Ong, D. E., and Brady, R. N. 1973. In vivo studies on the introduction of the $4-t$-double bond of the sphingenine moiety of rat brain ceramides. J. Biol. Chem. 248:3884-3888.

31. Radin, N. S. 1984. Biosynthesis of the sphingoid bases-a provocation. J. Lipid Res. 25:1536-1540.

32. Yoo, H-S., Norred, W. P., Wang, E., Merrill, A. H., Jr, and Riley, R. T. 1992. Fumonisin inhibition of de novo sphingolipid biosynthesis and cytotoxicity are correlated in LLC-PK1 cells. Toxicol. Appl. Pharmacol. 114:9-15.

33. Sugita, M., Williams, M., Dulaney, J. T., and Moser, H. W. 1975. Ceramidase and ceramide synthesis in human kidney and cerebellum. Description of a new alkaline ceramidase. Biochim. Biophys. Acta 398:125-131.

34. Schmid, P. C., Zuzarte-Augustin, M. L., and Schmid, H. H. O. 1985. Properties of rat liver $\mathrm{N}$-acylethanolamine amidohydrolase. J. Biol. Chem. 260:14145-14149.

35. Devane, W. A., Hanus, L., Breuer, A., Pertwee, R. G., Stevenson, L. A., Griffin, G., Gibson, D., Mandelbaum, A., Etinger, A., and Mechoulam, R. 1992. Isolation and structure of a brain constituent that binds to the cannabinoid receptor. Science 258:19461949.

36. Harzer, K., Paton, B. C., Poulos, A., Kustermann-Kuhn, B., Roggendorf, W., Grisar, T., and Popp, M. 1989. Sphingolipid activator protein deficiency in a 16-week-old atypical Gaucher disease patient and his fetal sibling: biochemical signs of combined sphingolipidoses. Eur. J. Pediatr. 149:31-39.

37. Bielawska, A., Linardic, C. M., and Hannun, Y. A. 1992. Modulation of cell growth and differentiation by ceramide. FEBS Lett. 307:211-214.

38. Abe, A., Wu, D., Shayman, J. A., and Radin, N. S. 1992 Metabolic effects of short-chain ceramide and glucosylceramide on sphingolipids and protein kinase C. Eur. J. Biochem. 210:765773.

39. Shayman, J. A., Mahdiyoun, S., Deshmukh, G., Barcelon, F., Inokuchi, J., and Radin, N. S. 1990. Glucosphingolipid dependence of hormone-stimulated inositol trisphosphate formation. J. Biol. Chem. 265:12135-12138. 